\title{
The Advantages of Not Entangling Macroscopic Diamonds at Room Temperature
}

\author{
Mark E. Brezinski ${ }^{1,2,3}$ \\ ${ }^{1}$ Center for Optical Coherence Tomography and Modern Physics, Department of Orthopedic Surgery, \\ Brigham and Women's Hospital, 75 Francis Street, MRB-114, Boston, MA 02115, USA \\ ${ }^{2}$ Center for Optical Coherence Tomography and Modern Physics, Department of Orthopedic Surgery, \\ Harvard Medical School, 25 Shattuck Street, Boston, MA 02115, USA \\ ${ }^{3}$ Department of Electrical Engineering and Computer Science, Massachusetts Institute of Technology, \\ Room 36-360, 77 Massachusetts Avenue, Cambridge, MA 02139, USA
}

Correspondence should be addressed to Mark E. Brezinski, mebrezin@mit.edu

Received 6 August 2012; Revised 2 December 2012; Accepted 12 December 2012

Academic Editor: Alan Migdall

Copyright ( 2012 Mark E. Brezinski. This is an open access article distributed under the Creative Commons Attribution License, which permits unrestricted use, distribution, and reproduction in any medium, provided the original work is properly cited.

\begin{abstract}
The recent paper entitled by K. C. Lee et al. (2011) establishes nonlocal macroscopic quantum correlations, which they term "entanglement", under ambient conditions. Photon(s)-phonon entanglements are established within each interferometer arm. However, our analysis demonstrates, the phonon fields between arms become correlated as a result of single-photon wavepacket path indistinguishability, not true nonlocal entanglement. We also note that a coherence expansion (as opposed to decoherence) resulted from local entanglement which was not recognized. It occurred from nearly identical Raman scattering in each arm (importantly not meeting the Born and Markovian approximations). The ability to establish nonlocal macroscopic quantum correlations through path indistinguishability rather than entanglement offers the opportunity to greatly expand quantum macroscopic theory and application, even though it was not true nonlocal entanglement.
\end{abstract}

\section{Introduction}

The ability to observe and control nonlocal macroscopic quantum coherence/correlations, under ambient conditions, would likely have a powerful influence across a wide range of fields. This was achieved recently by Lee et al., in Science, establishing phonon field quantum correlations in two spatially separated diamonds $[1,2]$. The paper was entitled entitled "Entangling Macroscopic Diamonds at Room Temperature." Two other studies nonlocally correlating reflectors (by our group) and a cesium gas respectfully support the results $[3,4]$. However, we will demonstrate on several grounds that while quantum correlations are established between the diamonds, they are not true entanglement.

The work in the Lee et al. paper is essentially a twoarm extension of the DLCZ (Duan, Lukin, Cirac, and Zoller) experiments [5-9]. Figure 1 is a schematic of the key components of the Lee experiment, but a more detailed schematic can be found in Figure 1 of the original paper. An ultrashort pulsed source is used whose outputs can be represented by a collection of single photon wavepackets (each wave packet can only interfere with itself), as they are neither entangled photons nor significant biphoton wavepackets. An MZI interferometer is used where diamonds are present in each arm which contain nearly identical Raman scatterers. The diamonds are $15 \mathrm{~cm}$ apart making any interaction between them macroscopic. The optical phonon modes of the diamond allow relatively low decoherence at room temperature because they have very high oscillatory frequencies $(40 \mathrm{THz})$ so are not readily disturbed by thermal energies. A pump pulse is sent through the interferometer of sufficient intensity to entangle with and stimulate the Raman scatterers. A Stokes photon is then emitted, with the diamond and Stokes photon entangled until detection. The extra energy remaining in the diamond (lost from the photon) is in the form of increased phonon field energy levels. If the detector registers one Stokes photon, it could have come from either of the diamond crystals in which one phonon was excited. This 
will be discussed in more detail below, but because the paths are indistinguishable, the system behaves as if the photon at the beam splitter came from both arms. Paraphrasing Dirac, a single photon wavepacket can only interfere with itself. Therefore, prior to the pump photon being detected, both phonon fields are stimulated. To confirm these results, a probe photon is introduced into the interferometer that interacts with the diamonds producing the anti-Stokes photons. The probe photon must interact with the diamond prior to the Stokes photon being detected. The nature of the detection scheme for the anti-Stokes photon allows determination if one or both phonon fields are stimulated. If we were only looking at one arm, prior to the Stokes photon detection, there is an entanglement between the Stokes photon, phonon field, and anti-Stokes photon. This is somewhat analogous to the nonlocal entanglements in the well-known studies performed by Brune et al. described below which we will use to support our conclusions about the Lee paper [10-12]. The key point of the Lee et al. paper made below is that the two phonon fields are quantum correlated, but not truly entangled as stated in the original paper.

Our analysis is that Lee's explanation, in the Science paper, for the quantum correlations generated between diamonds (resulting from the pump photons) is unlikely representative of the actual situation. They postulated a nonlocal entanglement between the diamonds. While we agree that quantum correlations are established, we do not believe that the data or analysis of the experimental design supports true entanglement. The essential points will be made here but the remainder of the paper will expand on these points. First, our examination supports that these nonlocal quantum correlations occur from a combination of paths indistinguishability (for a single photon wavepacket) plus nearly identical local entanglements (Raman scatterers) in each path [13-19]. The source is coherent so building the pulses up from single photon wavepackets (a photon can only interfere with itself) is a useful approach for illustrating the physics. The correlations between diamond phonons do not fit definitions of entanglement laid out, for example, by von Neumann, EPR-B, or GHZ [20-23].

Second, the pump photon/diamond interactions do not (and must not) meet the Born (system-environment coupling weak) or Markovian (memory effects of the environment are negligible) approximations of decoherence theory $[15,17]$. This occurs largely from the high frequency of the optical phonons and the strong coupling associated with the Raman scatterers. The results then of the pump photon/diamond interactions are more analogous to single photon wavepacket decoherence theory than nonlocal entanglement (point 1). Environmental interactions are occurring with indistinguishable paths, but in the case of the Science paper, coherence is expanded rather than lost (point 2) [1517]. This demonstrates perhaps the most important point of the paper, that the diamonds can lead to either decoherence (distinct local entanglements and meeting BornMarkov approximations) or coherence expansion (nearly identical local entanglements and not meeting Born-Markov approximations) depending on the setup.
In the next several paragraphs, the topics addressed will be as follows. First, nonlocal correlations will be examined, which can be represented by entangled states or states generated by indistinguishable paths. Second, we review the general definition of entanglement demonstrating why the nonlocal phonon field correlations in the Lee study are not accurately described as being entangled. Third, we discuss that path indistinguishability and the quantum correlations that can be generated. This and the previous paragraphs draw heavily from the work by pioneers that include von Neumman, Mandel, and Shih, as well as insights from recent decoherence theory by Zurcek and Zeh [15-17]. Decoherence theory is particularly useful in illustrating the point of this paper as indistinguishable paths lead to coherence while typical environmental entanglements generally lead to decoherence (with this paper representing an exception). Finally, we will also discuss how the authors represented visibility, concurrence, density operators, and statistical significance (particularly the correlation coefficient), and how these are completely consistent with nonlocal correlations from either indistinguishable paths or entanglement. We do not believe there is a basis to employ a two-mode squeeze state as discussed by Julsgaard et al., for the nonlocal correlations [4]. The appendix will speculate on the role misunderstanding type II SPDC sources and Dirac notation play in the misuse of the term "entanglement."

\section{Nonseparable States (Unfactorizable) and Quantum Correlations}

In order to discuss quantum correlations, including entangled states and those from path indistinguishability, density operators and their nonseparability will be discussed. The density operator is a Hermitian operator acting on Hilbert space with nonnegative eigenvalues whose sum is 1 (it is not a classical statistical operator). It should not be confused with a classical statistical matrix and it has its greatest value in calculating expectation values of physical properties [24]. A density operator does not specify a unique microscopic configuration, which is not surprising based on its definition and contains the information about superpositions between subsystems. Quantum correlations imply unfactorizable density operators between multiple entities, with quantum entanglement being one type. They demonstrate correlations that exceed those describable by classical mechanics. They can be local or nonlocal, with the latter used extensively in decoherence theory. For simplicity, in this paper, we will approximately describe the coherent portion of the system as the principal and everything else as the environment. We describe the principal as being represented by a pure state density operator, a single vector in Hilbert space (there is no loss of generality as a mixed state can be modeled using purification) [15]. In the Lee system, for clarity, the phonon fields are part of the principal and can be viewed as pure. But as the phonon fields are part of the diamond, the diamond itself is of low purity as the principal only makes up a small portion of the diamond.

Described more formally below, a state describing a pair of nonlocal quantum correlated entities (photons or 


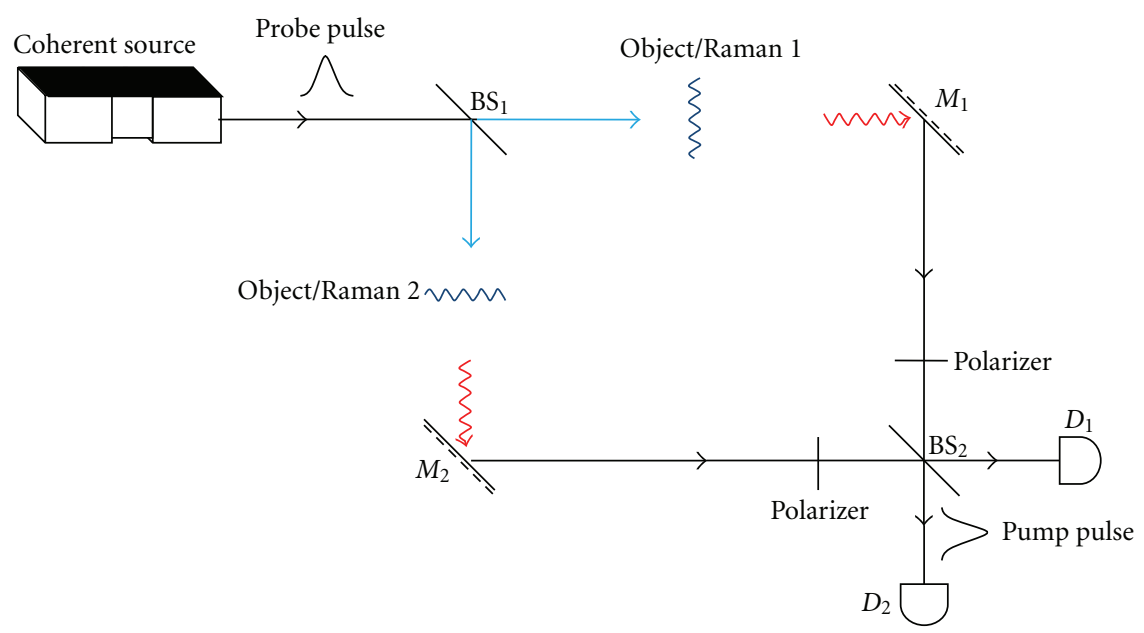

Figure 1: This diagram is a simplified version of the interferometer used in the Lee et al. experiments. Components have been removed which are needed for practical application but not for understanding the physical principles.

phonons) has an unfactorizable density operator for the pair that progresses forward in time via linear unitary operators. But in performing the trace operation to obtain the subsystems (e.g., a given diamond phonon field), these subsystems are represented by reduced density operators that move forward in time, unlike the true principal, via nonlinear unitary operators (i.e., the trace gives information on the subsystem statistical averages but is not the complete description of the subsystems) $[15,25]$. So for the Lee system, the principal contains both phonon fields that have inseparable density operators.

\section{Entanglement}

Entanglement, a type of quantum correlation, is a function of superposition and the linearity of Schrödinger's equation, but not generally path indistinguishability (which will be dealt with in a subsequent section) [22]. Here we will limit the discussion to complete entanglement and partial entanglement can be extrapolated from the discussion. Demonstrating interference with entangled photons though does require path indistinguishable (see the Appendix). The entanglement process is described by (as per von Neumman) [26] as follows:

$$
|\psi\rangle\left|a_{r}\right\rangle=\left(\sum_{i} c_{i}\left|s_{i}\right\rangle\right)\left|a_{r}\right\rangle \longrightarrow|\Psi\rangle=\sum_{i} c_{i}\left|s_{i}\right\rangle\left|a_{i}\right\rangle .
$$

This is a form which would be used to describe decoherence (or a one-arm Lee experiment) where the principal is given by the wavefunction $(\psi)[15,16]$. For two-particle entanglement, the wavefunction is simply replaced by a particle symbol. The arrow describes the unitary transform. The principal is represented in terms of the basis $s_{i}$ while the basis for the environment is given by $a_{r}$. Entanglement represents pairing of the eigenstates. It can be stated in an equivalent form that their conjugate pairs (e.g., position momentum) are completely correlated. So we have two points: (1) with two entangled particles, the two base states $s_{i}$ and $a_{i}$ develop a constant relationship; this is the core to entanglement. Measuring one of an entanglement pairs establishes the eigenvalue of both exactly from the superposition. (2) This point will be more clear from the path indistinguishability discussion, but without further interactions, entangled particles continue to have inseparable density operators. This is not true for quantum correlations from path indistinguishability where the inseparability is dependent on such factors as detector time and wavepacket width. We will use the phrase "conditionally inseparable." (3) The initial entanglement generally requires local interaction between atomic/subatomic particles, but can become nonlocal with entanglement swapping, for which we use for illustration the well-known-Brune studies described below $[10,12]$. This local-to-nonlocal entanglement can be found both in the Lee and Brune papers.

Equation (1) in the Lee paper (which is a DCLZ equation or one arm of the Lee interferometer) presents the initial local type of entanglement, in the annihilation operator form. This form was introduced by Dirac and expanded upon by Glauber for the quantum theory of light [27, 28]. The equation is

$$
\left|\Psi_{S}\right\rangle \approx\left[1+\varepsilon_{S} s^{+}\left(l_{S}\right) b^{+}\left(l_{S}\right)\right]|\mathrm{vac}\rangle .
$$

The equation is described in detail in the Lee paper. The essential point is that, for the potential, annihilation operators for the Stokes and phonon modes are in an inseparable product form. It will be seen that this is in contrast to (2) from that paper which is a superposition (below).

As noted, in addition to the Lee study being an extension of the DLCZ experiments, it is analogous to the pioneering experiments by Brune, entangling atoms with fields (and then a second atom) [10-12]. These studies are more useful than the Lee study for understanding the physics of entanglement and entanglement swapping because of the complex design of the Lee study. Its analogy is to a single arm of the 
Lee experiment. Rubidium atoms in a Rydberg state were passed through an EM field in the large $Q$ cavity. The atom and field become and remained entangled even after the Rubidium atom exited the system (i.e., until a measurement is made at the output of the device). One can then only speak of the combined Rubidium atom- $Q$ cavity field system as a pure state, which is non-local (this is analogous to the pump-phonon entanglement in the Lee experiment). The nonlocality can be extended even further by sending a second atom after the first (analogous to the probe photon in the Lee study). Here, the second atom becomes non-locally entangled with the first atom (which had already passed through) with perfect correlation (inseparable biparticle wave packet). The second atom non-local entanglement represents entanglement swapping with the field, which is no longer entangled. This demonstrates true nonlocal entanglement of the two atoms as the eigenstates of each, even though passing through the cavity at different times, exactly correlate. The two atoms of course are analogous to the Stokes and anti-Stokes photons in the Lee study and the EM field to the phonon field, except only one arm is used.

For a more formal description of entanglement and its subsystems, we will provide the mathematical framework for one EPR-B particle state. There are two observers of these particles, $A$ and $B$, separated by a large distance. One of these two entangled qubits is directed at each observer. The specific paths of each are inconsequential as long as no measurement has occurred. Neither does the order of detection nor the times between detection (as opposed to correlations from indistinguishable paths) for these entangled states. The Bell state used here is given by (let them be spin $1 / 2$ particles, with two states, 0 and 1 )

$$
\left|\Phi^{+}\right\rangle=|\Psi\rangle=\frac{1}{\sqrt{2}}\left(|0\rangle_{A} \otimes|0\rangle_{B}+|1\rangle_{A} \otimes|1\rangle_{B}\right)
$$

(Analogous Bell states with entangled energy and spin generated by a SPDC source type II, and the limitations, are discussed in the appendix.) Equation (3) is a true entangled state (spin superposition) in that the result of one observer exactly correlates with the results obtained with the second observer (irrespective of what spin axis is measured); the information of the system is complete. The density operator is given by

$$
\begin{aligned}
\hat{\rho}_{T} & =|\Psi\rangle\langle\Psi|=\hat{\rho}_{A} \otimes \hat{\rho}_{B} \\
& =\frac{|00\rangle\langle 00|+| 11\rangle\langle 00|+| 00\rangle\langle 11|+| 11\rangle\langle 11|}{2} .
\end{aligned}
$$

The density operator product is nonfactorizable. If we examine a subsystem, it is an inseparable state as the trace operation of each observer (here, observer $B$ ) yields less information than the whole

$$
\begin{aligned}
\rho^{A}= & \operatorname{Tr}(\rho) \\
= & \frac{\operatorname{Tr}_{B}(|00\rangle\langle 00|)+\operatorname{Tr}_{B}(|11\rangle\langle 00|)}{2} \\
& +\frac{\operatorname{Tr}_{B}(|00\rangle\langle 11|)+\operatorname{Tr}_{B}(|11\rangle\langle 11|)}{2} \\
= & \frac{|0\rangle\langle 0|\langle 0 \mid 0\rangle+| 1\rangle\langle 0|\langle 1 \mid 0\rangle}{2} \\
& +\frac{|0\rangle\langle 1|\langle 0 \mid 1\rangle+| 1\rangle\langle 1|\langle 1 \mid 1\rangle}{2} \\
= & \frac{|0\rangle\langle 0|+| 1\rangle\langle 1|}{2}=\frac{I}{2} .
\end{aligned}
$$

A reduced density operator is generated by the trace operation representing an improper mixed state, losing information about coherences. It is an expectation value. To paraphrase Schrödinger, the best possible knowledge of a whole does not include the best possible knowledge of its parts (if that knowledge is even available) [22]. In other words, the principal is inseparable as any description of the subsystem is incomplete as demonstrated by (5). We will contrast this true entanglement with correlations from indistinguishable paths where they are inseparable within certain experimental limits (e.g., path lengths and detector integration time).

\section{Path Distinguishability and First-Order Correlations}

Path indistinguishability can lead to nonlocal macroscopic correlations but generally not entanglement. A more complete discussion of coherence and indistinguishability can be found in the pioneering work of Mandel [20], reviewed by Shih (for both single- and two-photon (boson) correlations) $[13,19]$. The topic will be addressed here briefly. It should also be noted that our group in a previous paper also established nonlocal macroscopic correlations. Correlations were produced between two reflector arms with path indistinguishability using a thermal source under ambient conditions [3].

We begin looking at path indistinguishability for a single photon entering a beam splitter with the two arms as exit ports (essentially equivalent to the pump photon in the Lee paper). All first-order interference is a singlephoton wavepacket interference (as per Dirac), no matter what the intensity, along indistinguishable paths. Secondorder correlations are generally the interference of biphoton wavepackets and are reviewed elsewhere [3, 13, 19]. Firstorder coherence (single-photon wavepacket interference) has a wavefunction given by

$$
|\psi\rangle=\alpha|1\rangle_{1}|0\rangle_{2}+\beta|0\rangle_{1}|1\rangle_{2}
$$

Here the subscripts 1 and 2 are the two paths and the value in the ket represents occupation number. The alpha 
and beta terms take into account beam splitter ratios. Note that this is the form of (2) of the Lee paper and is not an entangled state. Equation (2) in the Lee paper was

$$
E\left|\Psi_{S}\right\rangle=\left[b_{L}^{+}\left(l_{S}\right)+e^{-i \varphi_{x}} b_{R}^{+}\left(l_{S}\right)\right]\left|\operatorname{vac}_{\mathrm{vib}}\right\rangle .
$$

Again, the specifics can be found in the original paper but unlike (7), the annihilation operators of the potential are now in a summation form rather than a product form.

Returning to (6), the density operator (in its expanded form) is given by

$$
\begin{aligned}
\hat{\rho}= & |\alpha|^{2}|1\rangle_{1}|0\rangle_{22}\left\langle\left. 0\right|_{1}\left\langle\left. 1|+| \beta\right|^{2} \mid 0\right\rangle_{1} \mid 1\right\rangle_{22}\left\langle\left. 1\right|_{1}\langle 0|\right. \\
& +\left[\alpha \beta *|1\rangle_{1}|0\rangle_{22}\left\langle\left. 1\right|_{1}\langle 0|+\text { h.c. }\right] .\right.
\end{aligned}
$$

The first two terms, the diagonal terms, are the DC terms that reduce fringe visibility to a maximum of $50 \%$ unless they can be removed (for true entanglement, there are no DC terms and maximum visibility is $100 \%$ ). When paths are distinguishable, these are the only nonzero terms. The third and fourth terms represent indistinguishable paths and generate interference (h.c. is the Hermitian conjugate or adjoint) (see Figure 3 in the Lee paper, as off-diagonal elements are not exclusive to entanglement as suggested). These off-diagonal elements are complex. It is important to note that the density operator is inseparable only within the constraints of path indistinguishable (e.g., wavepacket width, detector time, path lengths, etc.). Coherence time is an example. For an optical pulse, delay times must be within the coherence time. In contrast, for most entangled states, coherence time is not an issue except when demonstrating interference.

Young's interferometer is useful for illustrating the concepts of path indistinguishability. We will use diamonds similar to the Lee experiment before each slit in the Young's interferometer. Examining the Young's interferometer (Figure 2), if one or the other slit is blocked, the photons are registered on the screen with no interference pattern (NI). If both slits are open, classically it is easy to appreciate when waves pass through the apparatus, and an interference pattern will develop on the screen (I). The sinusoidal peaks in the Young's design are position-dependent interference on the screen (I) due to varying phase relationships. The Young's experiment results hold for a high intensity photon beam, but the interference is still single-photon wavepacket interference. Even when only one photon (or other particle) is coming from the source at a time, a first order interference pattern develops on the detection screen, which is predicted naturally from quantum mechanics but is unexplainable by classical mechanics (which would predict the NI pattern) $[13,14]$. This is because quantum mechanics is predicting the interference of potentials (along indistinguishable paths), and not intensities, as long as no measurement is made prior to the screen. There is no measurement of the pump photons in the Science paper until after the second beam splitter, so paths are still indistinguishable (in spite of the frequency shifts from the Raman scattering). So two-pump "beams" do not actually interfere as in the classical description of

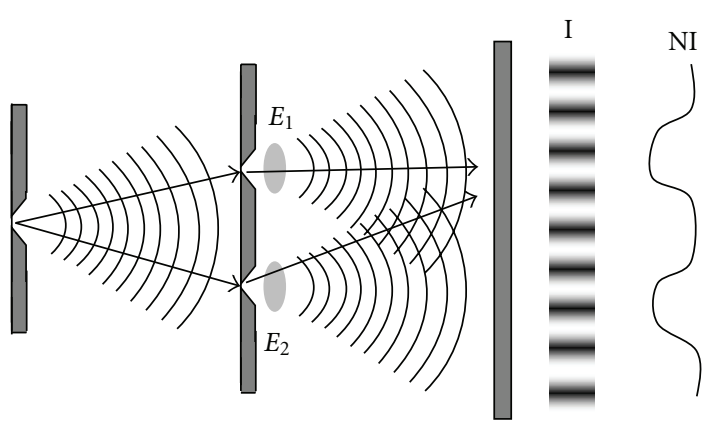

FIGURE 2: Illustration path indistinguishability and the influence environmental entanglements (diamonds) with Young's interferometer. The I is an interference pattern and the NI is no interference pattern. $E_{1}$ and $E_{2}$ are the diamonds.

interference after the second beam splitter; it is a singlephoton interference. Interference of indistinguishable paths potentials (of single-photon wave packets) leads to the interference. Interference is possible when these single photon potential paths are identical with respect to the diamond interactions, as is more formally described in the next paragraph. Quantum correlations are established between the diamonds because they are part of each indistinguishable path that led to the single photon interference.

Now we extend (8) beyond one photon (increase intensity) and include interactions with the environment, $E$ (diamonds) in the form of an inner product. This is a relatively common procedure for describing basic decoherence $[15$, 17], where the relevance to the diamond experiment will become apparent (though coherence is expanded rather than reduced). The interference pattern at the screen (of the Young's interferometer) is described by the cross terms (offdiagonal) in the density operator (it is in the expanded matrix form) as

$$
\begin{aligned}
\hat{\rho}=\frac{1}{2}\{\mid & \left.\psi_{1}\right\rangle\left\langle\psi_{1}|+| \psi\right\rangle_{2}\left\langle\left.\psi\right|_{2}\right. \\
& \left.+\left|\psi_{1}\right\rangle\left\langle\psi_{2}\left|\left\langle E_{2} \mid E_{1}\right\rangle+\right| \psi_{2}\right\rangle\left\langle\psi_{1}\right|\left\langle E_{2} \mid E_{1}\right\rangle\right\},
\end{aligned}
$$

where

$$
\begin{array}{ll}
\left|\psi_{1}\right\rangle\left\langle\psi_{1}\right|=\rho_{11}, & \left|\psi_{2}\right\rangle\left\langle\psi_{2}\right|=\rho_{22}, \\
|\psi\rangle_{1}\left\langle\left.\psi\right|_{2}=\rho_{12},\right. & |\psi\rangle_{2}\left\langle\left.\psi\right|_{1}=\rho_{21} .\right.
\end{array}
$$

The first two terms are again DC terms and the second two represent interference terms. The wavefunction (in the bras-kets) incorporates all properties of the photons (polarization, bandwidth, photon numbers, etc.) now and not just occupation number. As can be seen from the density operator, the interference pattern is independent of whether the photons come individually or at high intensity (if one of the wavefunctions was zero, interference would still occur). In the density operator equation, 1 and 2 correspond to the two potential paths the photon can take. The density operator contains an inner product $(E)$ in the last two terms 
that represents the diamonds which can be identical or distinct. The event that occurs at the screen is analogous to detection at $D_{3}$ in the Science paper.

To illustrate the counter-intuitive interaction of the photons and phonons leading to indistinguishable paths and coherence, Young's experiment will be examined by varying the Raman scattering. As a basic rule of quantum mechanics, which can be found in any introductory quantum mechanics textbook, until a measurement is made potentials are added then squared but once a measurement occurs, intensities (squared potentials) are added. If we initially ignore the $E$ terms (environmental entanglements/diamonds), the pattern on the screen demonstrates interference that comes from the last two terms (off-diagonal) of the density operator (again, even if one photon is coming through at a time). Now, if $E_{1}$ and $E_{2}$ are substantially different terms (inner product near zero), such as when the Stokes photons are of different frequencies, the third and fourth terms disappear as the paths become distinguishable. Interference is lost in this simple example of environmentally induced decoherence by Raman scattering [15-17]. The similarity of the Raman scattering in each arm affects the degree to which coherence (and interference) is lost (fringe visibility). If $E_{1}$ and $E_{2}$ are similar (inner product 1) such that Stokes photons are identical from the prospective of detection, the paths are indistinguishable even though the interaction with the diamonds occurred (and changed the frequency), and the interference pattern is maintained. The key point is that indistinguishablity is needed at the time of measurement (the detector).

But another critical point is that the Born and Markovian approximations are not met hence decoherence will result. The Born approximation is that the diamond-principal interaction is sufficiently weak and environment (diamond) large such that the principal does not significantly change the diamond. Obviously the coupling is strong (Raman scattering) and the diamond changes significantly (change in phonon frequency). The Markovian approximation, having no memory effects, means that self-correlations within the diamond/environment decay for all practical purposes instantly into the environment. If these two are not met (along with the diamond interactions being identical), then the diamonds become part of the coherent system rather than a source of decoherence. Together, the indistinguishable paths of single-photon coherence, near identical nature of Raman scattering, and not meeting the Born/Markovian approximations resulted in expansion of the coherence (the two diamonds become part of the principal, resulting in quantum correlations). This describes why the two phonon fields become correlated and why it does not require (or include) an explanation of true non-local entanglement between arms.

We suggest that confusion over the distinction between quantum correlations due to entanglement versus path indistinguishability has arisen, at least in part, over a misunderstanding of the type II spontaneous parametric downconversion (SPDC) source and overextending interpretations of Dirac notation, which is presented in the Appendix. This speculative topic is addressed in the Appendix.

\section{General Results of the Lee et al. Paper}

So to summarize, in the Lee paper the state, when using a single arm/diamond, is initially a Stokes-phonon(s) entanglement then Stokes-phonon(s)-anti-Stokes entanglement, arising from and remaining consistent with (1). It is an entanglement in the von Neumann sense as measurement of one subsystem exactly determines the state of the other subsystems. When two paths are used, the photon(s) and phonons are then entangled within a given path, but not entanglement of phonons between paths. However, the two diamonds are quantum correlated through path indistinguishability. The use of a coherent pulsed source allows the argument to be built up from single photon wavepacket interference.

As pointed out, the coherence expansion that results requires very specific conditions with respect to the diamonds. First, the high phonon frequency minimizes thermal decoherence. Second, the generated Stokes photons must be essentially identical with respect to detection. Third, the Born and Markovian approximations must not be met. Together, along with the path indistinguishability, this results in quantum correlations between the diamond phonons.

\section{Notes on the Probe Photons}

Just briefly discussing the probe photons, what is being measured is second-order correlation between detectors $D_{a+}$ and $D_{a_{-}}$, generated from phonon fields in the two arms, in a superposition. In general, we agree with the author's interpretations of the physical principles of the probe photons, which will not be reviewed here because of space limitations $[13,18,25]$. A quantitative description of these second-order correlations from both entangled photons and indistinguishable paths is best described in terms of the correlation functions, electric field operators, and annihilation operators. These are discussed elsewhere in detail for those interested $[13,19,27]$.

\section{Notes on the Quantitative Results}

The four-quantitative/qualitative results for discussion from the Science paper are the density operators presented, concurrence, confidence intervals, and visibility. (1) The density operators in Figures 3 and 4 of the Lee paper describe a coherent state as demonstrated by the offdiagonal coherences, which is not unique to entanglement. (2) There was some confusion in editorials/commentaries on the article that there was $98 \%$ concurrence. There was actually a $98 \%$ confidence interval that the concordance was positive (which as the reader is aware could mean it was $98 \%$ confidence the concurrence was extremely small but positive). The concurrence was positive and somewhere below $35 \%$, values consistent with quantum correlations that are not exclusive to an entangled state [29]. (3) The visibility graph (Figure 2 of the Science paper) demonstrates two main points. (A) The second order correlations are phase sensitive with opposite signs due to the beam splitter, which is known for second-order correlations. (B) The correlations between 
the pump and probe can exceed coincidence rates of classical correlations. These results demonstrate quantum correlations, but are not sufficient for specifically demonstrating quantum entanglement. This statement is also consistent with the experimental design analysis described above.

True entanglement between the phonon fields neither needs to be elicited as an explanation for the results nor leads to be proven in the paper. Though the phrase "entanglement of diamonds" attracts considerable attention, we believe that the establishment of quantum correlations/coherence between two macroscopic objects using path indistinguishability without nonlocal entanglements is far more important to the field. We point out that we have also achieved this with two macroscopic distant reflectors [3]. Path indistinguishability, under the local entanglement conditions described above, leads to quantum correlations. This approach required that no quantum source could be done under ambient conditions and potentially opens the door to a much larger number of applications than straight entanglement.

\section{Conclusion}

The recent paper in Science entitled "Entangling Macroscopic Diamonds at Room Temperature" by C. Lee et al. establishes nonlocal macroscopic quantum correlations between two diamonds. However, while the authors claim the correlations between diamonds represent entanglement, we present why a different underlying mechanism exists which explain the results. The quantum correlations are generated by path indistinguishability of first order correlations (single-photon wavepackets) in combination with essentially identical local entanglement in each arm. Irrespective, the results are of considerable importance. They offer a mechanism for generating macroscopic nonlocal quantum correlations under ambient conditions, which could represent a substantial advance to a wide range of applications.

\section{Appendix}

Unfortunately, many examples exist in the literature that treat quantum correlations from path indistinguishability and entanglement as essentially identical, an obstruction to the field and in part likely due to misunderstanding of the widely used SPDC II source (spontaneous parametric downconversion) and misuse of Dirac's notation. Two prominent examples are a 2008 Nature review on entanglement and the recent study claiming entanglement between two diamonds in Science $[1,30]$. A brief review of the SPDC may illustrate the point.

SPDC sources generally use a CW pumped nonlinear crystal to produce two energy entangled photon pairs (including entanglement of uncertainty) [31]. They were initially pursed to test EPR-B. Due to energy conservation, photon pairs' angular frequency and wave number are entangled. According to the standard theory of parametric downconversion, the two-photon state can be written as

$$
\begin{aligned}
|\Psi\rangle= & \int d \omega_{P} A\left(\omega_{P}\right) \\
& \times \int d \omega_{1} d \omega_{2} \delta\left(\omega_{1}+\omega_{2}-\omega_{P}\right) a^{+}\left(\omega_{1}\right) a^{+}\left(\omega_{2}\right)|0\rangle,
\end{aligned}
$$

where $\omega$ represents the angular frequency of the signal (1), idler (2), and pump (p) of the downconversion. The $a^{+}$ represents the respective annihilation operators. The delta function represents perfect frequency phase matching of the downconversion (i.e., entanglement). $A(\omega)$ is related to the wavepacket extent and is not critical to the discussion here (but it is when interfering entangled photons). This is a type I SPDC source (no fixed polarization relationship); note that the equation does not require path indistinguishability. With a type II SPDC source, the signal and the idler have orthogonal polarization states (i.e. the energy entangled photons are associated with perpendicular polarizations). The state is given by [32]

$$
\begin{aligned}
|\Psi\rangle= & \int d \omega_{P} A\left(\omega_{P}\right) \\
& \times \int d \omega_{1} d \omega_{2} \delta\left(\omega_{1}+\omega_{2}-\omega_{P}\right) a_{o}^{+}\left(\omega_{1}\right) a_{e}^{+}\left(\omega_{2}\right)|0\rangle .
\end{aligned}
$$

The subscripts on the signal and idler represent different polarization states associated with the entangled energy states $(o$ and $e)$. Again, the energy states are entangled (and thereby the polarization states) without any use of indistinguishable paths.

Now, using a SPDC II source with an interferometer (Figure 3) illustrates both entanglement and path indistinguishability. In this setup, prior to the beam splitter, the photons are both entangled by energy and polarization. After the beam splitter, indistinguishable paths are used to generate interference. Under the correct setup of the polarizers $\left(P_{1}\right.$ and $\left.P_{2}\right)$ in each arm, Bell states can be generated which can be used to test, for example, EPR-B. The path indistinguishability after the beam splitter does not cause the entanglement, but rather it is used to generate Bell states from the already entangled states. Authors often abbreviate the wave function for these Bell states, (entangled photons grouped by indistinguishable paths) for example, as $(1 / 2)(|\mathrm{HV}\rangle+|\mathrm{VH}\rangle)$. This representation, as seen in the Nature review, can be misleading, because it drops the energy/polarization entanglement that exists without the beam splitter, as well as the wavepacket for the biphoton (basically just using the $e$ and $o$ from (A.2) and giving the impression that they are being entangled by the beam splitter) [37]. Interfering light from the SPDC II source in the Nature paper, a common yet incorrect statement in Figure 2 of that paper is made, "However, in the regions where the two cones overlap, the state of the photons will be $|\mathrm{HV}\rangle+|\mathrm{VH}\rangle$. It is around these points that entangled photons are generated." This abbreviated representation of 


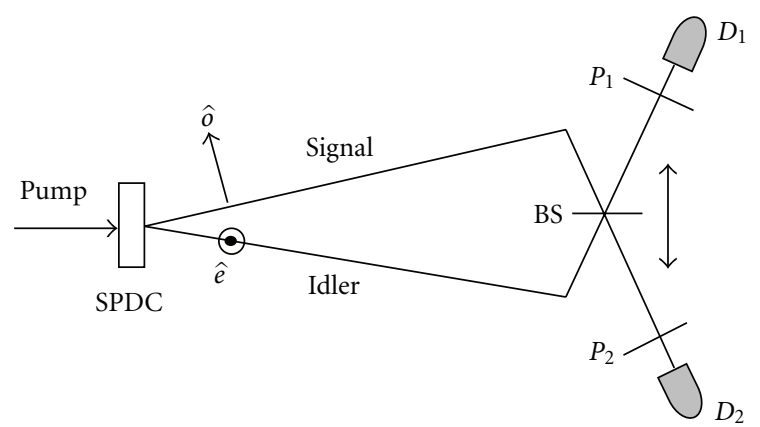

Figure 3: An SPDC type II source using a beam splitter used to generate Bell states.

the state ignores the already entangled energy/polarization in areas outside the overlap (9), as well as the space-time probability density. This leads to a misunderstanding of the physics. Entanglement exists in the areas outside the overlap, not just Bell states. Similarly, path indistinguishability did not lead to entanglement of diamonds in the Lee experiment.

The example also illustrates the misuse of Dirac notation, which seems particularly common in the quantum communication and computer fields. Dirac notation is a powerful shorthand technique for describing quantum information flow. But it is frequently treated as representing the state of a system, which it generally does not do. If we represent a vacuum and photon by $|01\rangle+|10\rangle$, this neither tells us, for example, about the state of the vacuum nor the bandwidth of the photon. But this is how it is often interpreted leading to erroneous conclusions.

\section{Acknowledgments}

This paper is sponsored by the National Institutes of Health, Contracts R01-AR44812, R01-EB000419, R01 AR46996, R01- HL55686, R21 EB015851-01, and R01-EB002638.

\section{References}

[1] K. C. Lee, M. R. Sprague, B. J. Sussman et al., "Entangling macroscopic diamonds at room temperature," Science, vol. 334, no. 6060, pp. 1253-1256, 2011.

[2] L. M. Duan, "Quantum correlation between distant diamonds," Science, vol. 334, no. 6060, pp. 1213-1214, 2011.

[3] M. E. Brezinski and B. Liu, "Nonlocal quantum macroscopic superposition in a high-thermal low-purity state," Physical Review A, vol. 78, no. 6, Article ID 063824, 13 pages, 2008.

[4] B. Julsgaard, A. Kozhekin, and E. S. Polzik, "Experimental long-lived entanglement of two macroscopic objects," Nature, vol. 413, no. 6854, pp. 400-403, 2001.

[5] L. M. Duan, M. D. Lukin, J. I. Cirac, and P. Zoller, "Longdistance quantum communication with atomic ensembles and linear optics," Nature, vol. 414, no. 6862, pp. 413-418, 2001.

[6] D. N. Matsukevich and A. Kuzmich, "Quantum state transfer between matter and light," Science, vol. 306, no. 5696, pp. 663666, 2004.

[7] K. S. Choi, H. Deng, J. Laurat, and H. J. Kimble, "Mapping photonic entanglement into and out of a quantum memory," Nature, vol. 452, no. 7183, pp. 67-71, 2008.
[8] C. W. Chou, H. De Riedmatten, D. Felinto, S. V. Polyakov, S. J. Van Enk, and H. J. Kimble, "Measurement-induced entanglement for excitation stored in remote atomic ensembles," Nature, vol. 438, no. 7069, pp. 828-832, 2005.

[9] T. Chanelière, D. N. Matsukevich, S. D. Jenkins, S. Y. Lan, T. A. B. Kennedy, and A. Kuzmich, "Storage and retrieval of single photons transmitted between remote quantum memories," Nature, vol. 438, no. 7069, pp. 833-836, 2005.

[10] M. Brune, E. Hagley, J. Dreyer et al., "Observing the progressive decoherence of the "meter" in a quantum measurement," Physical Review Letters, vol. 77, no. 24, pp. 4887-4890, 1996.

[11] M. Brune, S. Haroche, J. M. Raimond, L. Davidovich, and N. Zagury, "Manipulation of photons in a cavity by dispersive atom-field coupling: quantum-nondemolition measurements and generation of Schrödinger cat states," Physical Review A, vol. 45, no. 7, pp. 5193-5214, 1992.

[12] T. Meunier, S. Gleyzes, P. Maioli et al., "Rabi oscillations revival induced by time reversal: a test of mesoscopic quantum coherence," Physical Review Letters, vol. 94, no. 1, Article ID 010401, 4 pages, 2005.

[13] Y. Shin, An Introduction to Quantum Optics: Photon and Biphoton Physics, CRC Press, New York, NY, USA, 2011.

[14] L. Mandel, "Coherence and indistinguishability," Optics Letters, vol. 16, no. 23, pp. 1882-1883, 1991.

[15] M. Schlosshuaser, Decoherence and the Quantum to Classical Transition, Springer, Melbourne, Australia, 2007.

[16] W. H. Zurek, "Decoherence and the transition from quantum to classical," Physics Today, vol. 44, no. 10, pp. 36-44, 1991.

[17] M. A. Neilson and I. L. Chuang, Quantum Computer and Quantum Information, Cambridge University Press, Cambridge, UK, 2007.

[18] L. Mandel, "Quantum effects in one-photon and two-photon interference," Reviews of Modern Physics, vol. 71, no. 2, pp. S274-S282, 1999.

[19] H. Chen, T. Peng, S. Karmakar, Z. Xie, and Y. Shih, "Observations of anti-correlations in incoherent thermal light fields," Physical Review A, vol. 84, Article ID 033835, 2011.

[20] A. Einstein, B. Podolsky, and N. Rosen, "Can quantummechanical description of physical reality be considered complete?" Physical Review, vol. 47, no. 10, pp. 777-780, 1935.

[21] D. Greenberger, M. Horne, A. Shimony, and A. Zeilinger, "Bell's theorem without inequalities," American Journal of Physics, vol. 58, p. 1131, 1990.

[22] E. Schrödinger, "Die gegenwärtige situation in der quantenmechanik," Naturwissenschaften, vol. 23, no. 807, pp. 823-844, 1935.

[23] G. Jarger, Entanglement, Information, and the Interpretation of Quantum Mechanics, Springer, New York, NY, USA, 2009.

[24] K. Blum, Density Matrix Theory and Applications, Plenum Press, New York, NY, USA, 1996.

[25] C. K. Hong, Z. Y. Ou, and L. Mandel, "Measurement of subpicosecond time intervals between two photons by interference," Physical Review Letters, vol. 59, pp. 2044-2046, 1987.

[26] J. von Neumann, Mathematical Foundations of Quantum Mechanics, chapter 4, Princeton University Press, Princeton, NJ, USA, 1955.

[27] R. J. Glauber, "The quantum theory of optical coherence," Physical Review, vol. 130, no. 6, pp. 2529-2539, 1963.

[28] P. A. M. Dirac, "The question theory of the emission and absorption of radiation," Proceedings of the Royal Society of London A, vol. 114, no. 767, pp. 243-265, 1927.

[29] R. Hildebrand, "Concurrence revisted," Journal of Mathematical Physics, vol. 48, no. 10, Article ID 102108, 23 pages, 2007. 
[30] V. Vedral, "Quantifying entanglement in macroscopic systems," Nature, vol. 453, no. 7198, pp. 1004-1007, 2008.

[31] P. G. Kwiat, K. Mattle, H. Weinfurter, A. Zeilinger, A. V. Sergienko, and Y. Shih, "New high-intensity source of polarization-entangled photon pairs," Physical Review Letters, vol. 75, no. 24, pp. 4337-4341, 1995.

[32] Y. Shih, "Entangled Photons," IEEE Journal on Selected Topics in Quantum Electronics, vol. 9, no. 6, pp. 1455-1467, 2003. 

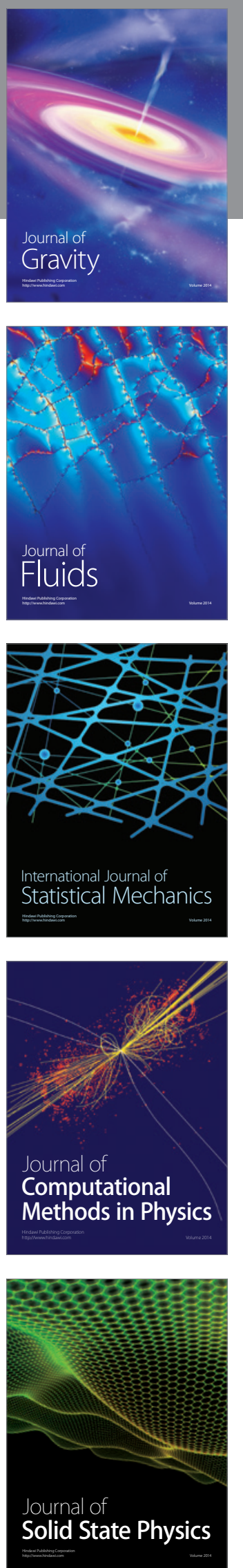

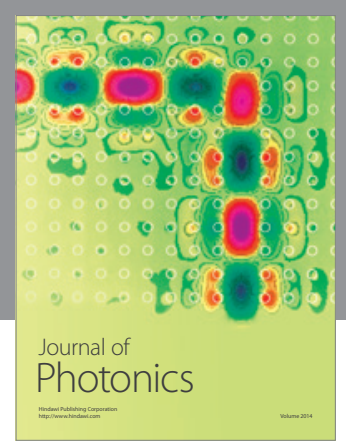

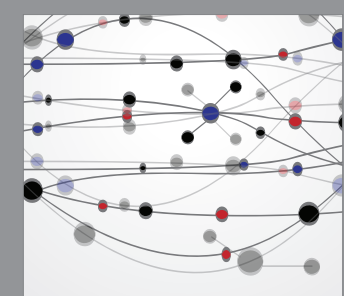

The Scientific World Journal
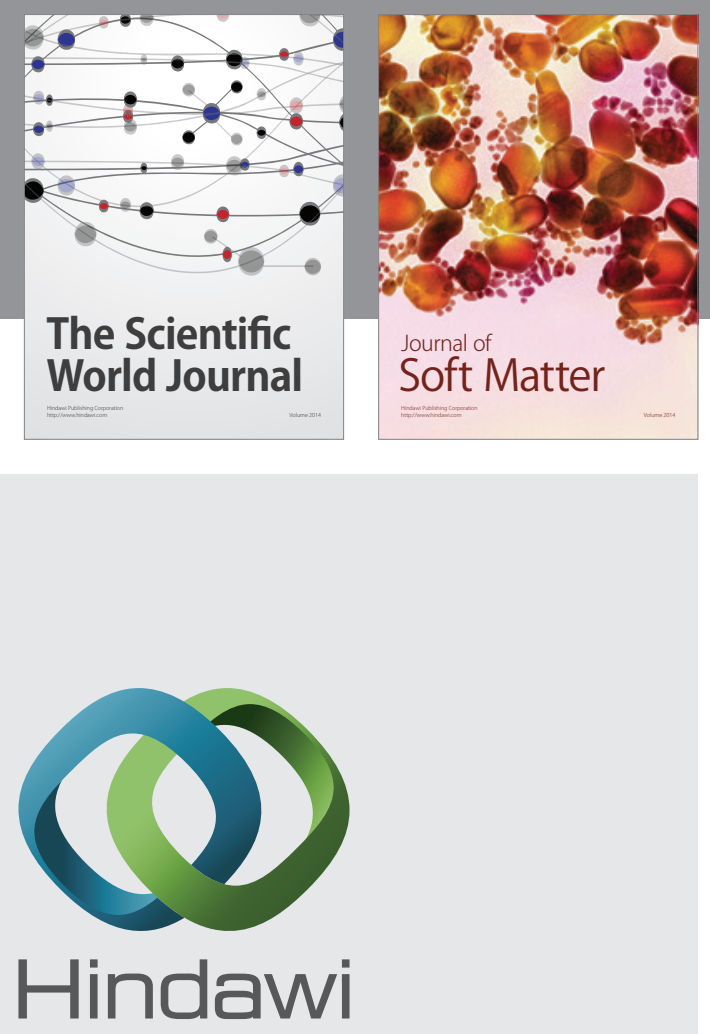

Submit your manuscripts at

http://www.hindawi.com
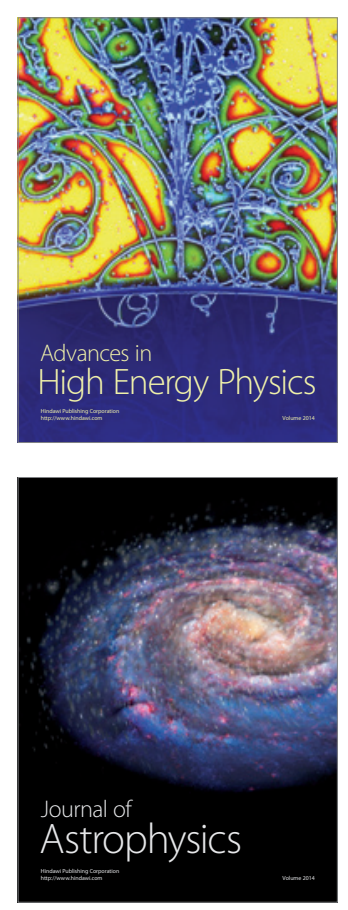
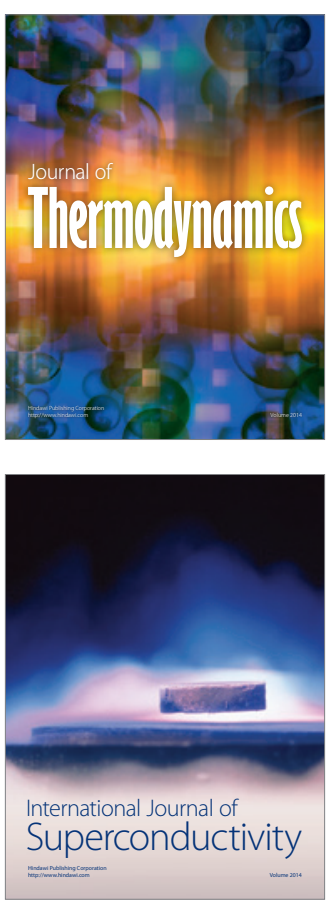
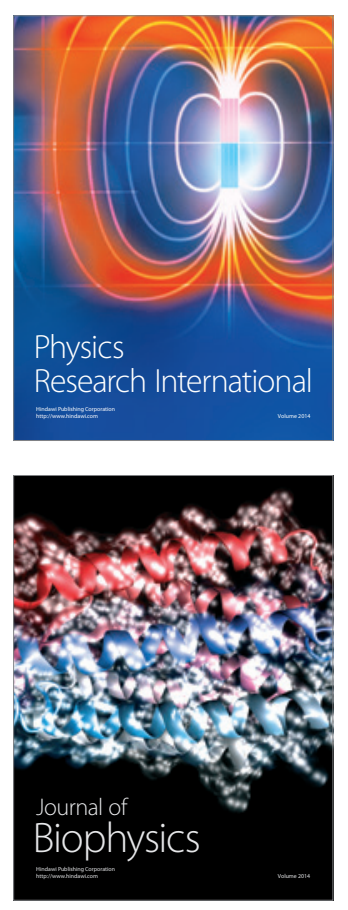
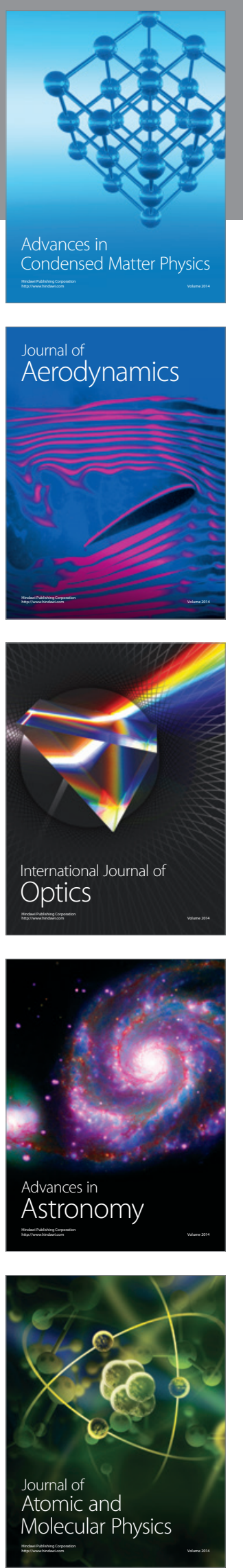